OPEN ACCESS

Edited by:

Sabina Passamonti,

University of Trieste, Italy

Reviewed by:

Xiaomin Liang,

Gilead, United States

Massimo Valoti,

University of Siena, Italy

*Correspondence:

Xue-Yan Wang

wangxueyan2018@163.com

Ji-Fu Wei

weijifu@hotmail.com

De-Yun Wang

entwdy@nus.edu.sg

${ }^{t}$ These authors have contributed equally to this work and share first

authorship

Specialty section:

This article was submitted to Drug Metabolism and Transport,

a section of the journal

Frontiers in Pharmacology

Received: 19 October 2019

Accepted: 28 February 2020

Published: 18 March 2020

Citation:

Shi H-Y, Pan C, Ma T-T, Chen Y-L,

Yan W-J, Liu J-G, Cao M-D,

Huang $H-D$, Wang $D-Y$, Wang $X-Y$ and Wei J-F (2020) Clinical Efficacy

Evaluation of 1-Year Subcutaneous

Immunotherapy for Artemisia sieversiana Pollen Allergic Rhinitis by

Serum Metabolomics.

Front. Pharmacol. 11:305.

doi: 10.3389/fphar.2020.00305

\section{Clinical Efficacy Evaluation of 1-Year Subcutaneous Immunotherapy for Artemisia sieversiana Pollen Allergic Rhinitis by Serum Metabolomics}

\author{
Hai-Yun Shi' ${ }^{1+}$, Chen Pan ${ }^{2,3 t}$, Ting-Ting Ma', Yan-Lei Chen ${ }^{1}$, Wei-Jun Yan ${ }^{4}$, Jian-Guo Liu, \\ Meng-Da Cao ${ }^{2}$, Hong-Dong Huang ${ }^{5}$, De-Yun Wang ${ }^{6 *}$, Xue-Yan Wang ${ }^{1 *}$ and Ji-Fu Wei ${ }^{2 *}$ \begin{abstract}
of Nanjing Medical University, Nanjing, China, ${ }^{3}$ School of Basic Medicine and Clinical Pharmacy, China Pharmaceutical University, Nanjing, China, ${ }^{4}$ Duolun People's Hospital, Inner Mongolia, China, ${ }^{5}$ Department of Nephrology, Beijing Friendship Hospital, Faculty of Kidney Diseases, Capital Medical University, Beijing, China, ${ }^{6}$ Department of Otolaryngology, Yong Loo Lin School of Medicine, National University of Singapore, Singapore, Singapore
\end{abstract} \\ 'Department of Allergy, Beijing Shijitan Hospital, Capital Medical University, Beijing, China, ${ }^{2}$ The First Affiliated Hospital
}

Subcutaneous immunotherapy is the only treatment that improves the natural progression of allergic rhinitis and maintains long-term outcomes after discontinuation of the drug. Metabolomics is increasingly applied in the study of allergic diseases, including allergic rhinitis. However, little is known about the discovery of metabolites that can evaluate clinical efficacy and possible mechanisms of Artemisia sieversiana pollen subcutaneous immunotherapy. Thirty-three patients with Artemisia sieversiana pollen allergic rhinitis significantly improved after 1-year subcutaneous immunotherapy treatment, while ten patients were ineffective. Pre- and post-treatment serum samples from these patients were analyzed by metabolomics based on the combined detection of liquid chromatography-mass spectrometry and gas chromatographymass spectrometry. As a result, L-Tyrosine can be a potential biomarker because of its opposite trend in effective patients and ineffective patients. And mechanism of immunotherapy may be closely related to $\mathrm{NO}$ and nitric oxide synthase. The discovery of potential biomarkers and metabolic pathways has contributed to the in-depth study of mechanisms of subcutaneous immunotherapy treatment of Artemisia sieversiana pollen allergic rhinitis.

Keywords: subcutaneous immunotherapy, clinical efficacy, seasonal allergic rhinitis, metabolomics, LC-MS, GC-MS

\section{INTRODUCTION}

Allergic rhinitis is a type I allergic disease of the nasal mucosa, characterized by paroxysmal repetitive sneezing, watery rhinorrhea, and nasal blockage (Okubo et al., 2017). Allergic rhinitis affects all age groups, increasing in prevalence globally (Wang et al., 2016). Recent studies showed that allergic rhinitis was thought to affect up to $10-40 \%$ of the worldwide population

Abbreviations: AIT, allergen-specific immunotherapy; FC, fold change; KEGG, Kyoto Encyclopedia of Genes and Genomes; OSC, orthogonal signal correction; OSC-PLS-DA, orthogonal signal correction partial least-squares discriminant analysis; PCA, principal component analysis; PLS-DA, partial least-squares discriminant analysis; RQLQ, rhinoconjunctivitis quality of life questionnaires; SCIT, subcutaneous immunotherapy; SIT, specific immunotherapy; TNSS, total nasal symptom scores. 
(Ma et al., 2014; Song et al., 2015; Bachmann and Kündig, 2017; Wang et al., 2017, 2018). The prevalence of allergic rhinitis in the United States was 19.9\% (Wallace and Dykewicz, 2017), while that in adults in Europe ranged from 17 to $28.5 \%$ (Brożek et al., 2017; Zheng et al., 2018). The results of a latest meta-analysis indicated that the incidences of allergic rhinitis were $15.79 \%$ in Chinese children and $13.26 \%$ in Chinese adult (Hu S. J. et al., 2017).

Allergic rhinitis is classified into perennial and seasonal allergic rhinitis. Pollinosis is seasonal allergic rhinitis caused by pollen antigens, frequently complicated by allergic conjunctivitis (Okubo et al., 2017). Artemisia sieversiana (Artemisia siversiana Ehrh. ex Willd.) pollen is one of the most common outdoor allergens in China, especially in the north of China. It is also one of the main causes of seasonal allergic rhinitis (Tang et al., 2015; Hu W. H. et al., 2017; Wang et al., 2018). Artemisia sieversiana pollen accounted for $36.7 \% \sim 50.5 \%$ of the pollen content from August to September in Beijing (Wang et al., 2017). A crosssectional study has shown that about $11.3 \%$ of patients in China with respiratory allergies were sensitized to Artemisia sieversiana pollen (Gao et al., 2019). This value was much higher in Northern China (78.6\%) (Cui and Yin, 2018).

During the summer and autumn pollen season of Artemisia sieversiana, seasonal allergic rhinitis of Artemisia sieversiana pollen brings inconvenience to patients, such as nasal itching, sneezing, rhinorrhea, nasal congestion, even inducing asthma (Brożek et al., 2017). Currently, the clinical treatment of seasonal allergic rhinitis includes non-specific therapy and SIT. Non-specific therapy mainly refers to drug treatment, such as antihistamines (H2 receptor antagonists), leukotriene receptor blockers, corticosteroids and so on (Greiner et al., 2011). There are two main forms of SIT: SCIT and sublingual allergenspecific immunotherapy (SLIT). However, it is difficult to improve the quality of life of patients and keep therapeutic control (Wallace and Dykewicz, 2017) for lifelong time through drug treatment. Only immunotherapy with individually targeted allergens can alter the natural history of allergic rhinitis (Jacobsen et al., 2007), improve symptom and eliminate the causes of disease, especially suitable for patients with severe or moderate seasonal allergic rhinitis (Cox et al., 2011; Greenhawt et al., 2017).

Subcutaneous immunotherapy was the first applied treatment method of SIT (Cox et al., 2011). During the clinical application for more than one century, SCIT has proved to be well safety under standardized regimens and the professional operation of professionals (Passalacqua et al., 2016). By repeatedly injecting a specific allergen extraction subcutaneously, the patient can reduce or eliminate allergic rhinitis symptoms, and has longterm effects after stopping the drug. SCIT acts by deeply affecting the immunologic allergen-oriented response at various levels (Noon, 1911). SCIT generally takes 3 years and can produce significant and stable symptom improvement after 1year treatment (Yukselen et al., 2012). The current indicators for the evaluation of SCIT efficacy are subjective indicators. Lack of recognized objective indicators makes the evaluation of SCIT efficacy have certain limitations. Simultaneously, the detailed mechanisms are not clear (Polosa et al., 2004).
Metabolomics is a powerful exploratory tool for discovering interactions between different biochemical molecules and pathways of disease or drugs (Omabe et al., 2018), advancing our understanding of disease progression and drug effects. It was also widely used for the study of allergic diseases. Chen et al. (2019) found that baicalin has protective effects on allergic rhinitis rats by inhibiting the release of immunoglobulin E, histamine, interleukin (IL)-1 $\beta$, IL-4, and IL- 6 by metabolomics studies. Zhuang et al. (2018) found that the potential metabolic pathways of Xanthii Fructus in treating allergic rhinitis in mice include glycerophospholipid metabolism and branchedchain amino acid metabolism. Immunotherapy often affects human metabolism, leading to metabolic disorders (Johnson et al., 2016). However, there are few reports identifying the potential mechanism of SCIT in the treatment of allergic rhinitis by metabolomics. In the present study, we tried to investigate metabolite changes and metabolic activities of Artemisia sieversiana pollen allergic rhinitis patients after SCIT and speculate related cellular signaling pathways and epigenetics by metabolomics.

\section{MATERIALS AND METHODS}

\section{Patients and Study Design}

Seventy-eight patients with Artemisia sieversiana pollen allergic rhinitis were recruited in Beijing Shijitan Hospital, Affiliated to Capital Medical University, from July 28 to 31, 2016. Inclusion criteria: course of disease more than 1 year. Typical symptoms appear in the summer and autumn pollen season, and asymptomatic or mild symptoms in the non-pollen season (non-pollen season VAS $<3$ ). Results of intradermal test of Artemisia sieversiana pollen allergen $\geq+++$, and sIgE $\geq$ II (Uni-CAP allergen-specific IgE detection system). Other types of allergen skin test negative, or " + " and above (including " + ") but specific IgE < II level (cat and dog allergens, even if the skin test and specific $\operatorname{IgE}$ do not meet the above conditions, as long as the family does not keep pets Can also be selected). Other sage pollen allergenspecific $\operatorname{IgE}$ is 2 or higher than wormwood pollen. Patients received SCIT treatment with standardized Artemisia sieversiana pollen allergen extraction purchased from Beijing MacroUnion Pharmaceutical Limited Corporation, Beijing, China (batch number: S20130001, total protein content $1.75 \mathrm{mg} / 5 \mathrm{~mL}$ ). Standard extraction process for allergens: buffered saline extract and raw materials are mixed to extract allergens, filtered by $\mathrm{pH}$, filtered, prepared, packaged, and tested to obtain the finished allergen extract prickly liquid. The main component of the pricking liquid is a soluble protein mixture in the pollen of Artemisia sieversiana. All the patients received standardized regimens and protocols, under operation of professionals in hospital. Patients' diaries were regularly completed, including TNSS, RQLQ, VAS scores, olfactory function grades and allergic conjunctivitis symptoms scores. The SCIT treatment lasted 1 year.

Thirty-two people fell off during the treatment. The remaining patients $(n=46)$ were evaluated for 1 -year efficacy 
based on comprehensive objective indicator. The curative effect was evaluated by symptoms and sign scores. The curative effect index $(\%)=$ (pre-treatment total score - posttreatment total score)/pre-treatment total score $\times 100 \%$ (Gu and Dong, 2005). Patients with effective therapeutic index ( $\geq 66 \%$, significantly improved, $n=33$ ) were included the effective group. Patients with ineffective therapeutic index ( $\leq 25 \%, n=10)$ were included the ineffective group and admitted as negative control. The remaining patients $(>25 \%,<66 \%$, not significantly effective or ineffective, $n=3$ ) were not included in the analysis because their treatment results were not clear.

\section{Ethics Statement}

The study protocol was in accordance with the ethical standards of the Declarations of Helsinki. The protocol of this study was approved by the Institutional Review Board of Beijing Shijitan Hospital, Affiliated to Capital Medical University, Beijing, China (Z161100000516006, November 2, 2015), and written informed consent was obtained from all subjects.

\section{Serum Sample Collection}

Serum samples pre-treatment and post-treatment were collected from both effective group $(n=33)$ and ineffective group patients $(n=10)$. Approximately $4 \mathrm{~mL}$ of peripheral venous blood of the patients was collected with EDTA anticoagulation tubes and immediately centrifuged at $1,500 \mathrm{rpm}\left(10 \mathrm{~min}, 4^{\circ} \mathrm{C}\right)$. Serum was separated by centrifugation, dispensed at $200 \mu \mathrm{L} /$ tube, and stored at $-70^{\circ} \mathrm{C}$ until serum metabolomics analysis. The samples needed to be processed within $1 \mathrm{~h}$ after separation from human body.

\section{Sample Preparation of Liquid Chromatography-Mass Spectrometry (LC-MS)}

Take $20 \mu \mathrm{L}$ of serum, add $225 \mu \mathrm{L}$ of ice methanol, vortex for 10 s. Add $750 \mu \mathrm{L}$ ice methyl tert-butyl ether (MTBE), vortex for $10 \mathrm{~s}$. Shake at $4^{\circ} \mathrm{C}$ for $10 \mathrm{~min}$. Add $188 \mu \mathrm{L}$ of ultrapure water, vortex for $20 \mathrm{~s}$. Centrifuge at $14,000 \mathrm{rpm}$ for $2 \mathrm{~min}$ at $4^{\circ} \mathrm{C}$. $350 \mu \mathrm{L}$ of the supernatant was pipetted into a $1.5 \mathrm{~mL}$ centrifuge tube, spin dried, and placed at $-20^{\circ} \mathrm{C}$ for testing. The remaining liquid was centrifuged for $2 \mathrm{~min}$ again, $125 \mu \mathrm{L}$ of the lower layer was aspirated, evaporated, and placed at $-20^{\circ} \mathrm{C}$ for testing. Add the upper layer of the sample to $110 \mu \mathrm{L}$ of methanol: toluene (9:1). Vortex for $10 \mathrm{~min}$, sonicate for $10 \mathrm{~min}$, centrifuge at $18,000 \mathrm{rpm}$ for $10 \mathrm{~min}$. The lower layer of the dried sample was vortexed with $60 \mu \mathrm{L}$ of acetonitrile: water (4:1) for $10 \mathrm{~min}$, sonicated for $10 \mathrm{~min}$, centrifuged at $14,000 \mathrm{rpm}$ for $10 \mathrm{~min}$. The positive ion mobile phase was $\mathrm{A}$ (acetonitrile: water $=60: 40,10 \mathrm{mmol}$ ammonium formate, $0.1 \%$ formic acid) and B (isopropanol: acetonitrile $=70: 30,10 \mathrm{mmol}$ of ammonium formate, $0.1 \%$ formic acid). The anion mobile phase was A (acetonitrile: water $=60: 40,10 \mathrm{mmol}$ ammonium acetate) and B (isopropanol: acetonitrile $=90: 10,10 \mathrm{mmol}$ ammonium acetate).

\section{Sample Preparation of Gas Chromatography-Mass Spectrometry (GC-MS)}

Add $30 \mu \mathrm{L}$ heptadecanoic acid $(0.3 \mathrm{mg} / \mathrm{mL})$ and $400 \mu \mathrm{L}$ methanol to $50 \mu \mathrm{L}$ serum, followed by vortexing and centrifugation at $13,000 \mathrm{rpm}$ for $10 \mathrm{~min}$. $300 \mu \mathrm{L}$ of the supernatant was taken and dried with nitrogen. Add $500 \mu \mathrm{L}$ of methoxyamine pyridine solution $(15 \mathrm{mg} / \mathrm{mL})$, react at $60^{\circ} \mathrm{C}$ for $2 \mathrm{~h}$. Add $60 \mu \mathrm{L} \mathrm{N,O-Bis(trimethylsilyl)trifluoroacetamide}$ (BSTFA) with $1 \%$ trimethylchlorosilane (TMCS), react at $70^{\circ} \mathrm{C}$ for $1 \mathrm{~h}$. The mixture was centrifuged at $13,000 \mathrm{rpm}$ for $10 \mathrm{~min}$ at $4^{\circ} \mathrm{C}$, and then the supernatant was taken for injection measurement.

\section{Data Pre-processing}

LC-MS and GC-MS spectra were manually phased, baselinecorrected and referenced to TSP at $0.0 \mathrm{ppm}$, using Bruker Topspin 3.0 software (Bruker GmbH, Karlsruhe, Germany) (Li et al., 2017; Hong et al., 2018). Then they were automatically exported to ASCII files using MestReNova (Version 8.0.1, Mestrelab Research SL), which were then imported into " $\mathrm{R}$ " software ${ }^{1}$ to do further phase and baseline correction and peak alignment with an in-house developed R-script (Li et al., 2017; Lv et al., 2017; Zhang et al., 2017). The one-dimensional (1D) spectra were automatically binned between 0.2 and $10 \mathrm{ppm}$ using a dynamic adaptive binning approach for subsequent statistical analysis (Wan et al., 2017). Exclude the regions of the residual water and affected signals. The remaining regions of each spectrum were normalized and mean-centered and Pareto-scaled before further multivariate statistical analysis (Chen et al., 2017).

\section{Multivariate Analysis}

In order to filter out irrelevant effects and maximize the discrimination between intergroup differences, unsupervised PCA and supervised OSC-PLS-DA were applied for multivariate statistical analysis (Xing et al., 2018). The OSC was a technique that applied prior to PLS-DA to filter out unrelated variables that were not concerning the class discrimination so as to minimize the influence of unrelated signals (Chen et al., 2017). To assess the validity of the established OSC-PLS-DA model, a repeated twofold cross-validation method and permutation test $(n=2000)$ were applied. The validity of the models against over fitting was assessed by the parameters R2Y, and the predictive ability was described by Q2Y (Li et al., 2017). Color-coded loadings plot and $S$-plot were constructed to reveal variables that contributed to the group separation. The observed statistic $p$-values via permutation testing which were less than 0.05 confirmed the significance of the OPLS-DA model at a $95 \%$ confidence level.

\section{Univariate Analysis}

Parametric Student's $t$-test and non-parametric MannWhitney test were performed to evaluate the differences of metabolites between groups. The FC values of the identified metabolites between groups as well as their associated $p$-values

\footnotetext{
${ }^{1}$ http://cran.r-project.org/
} 

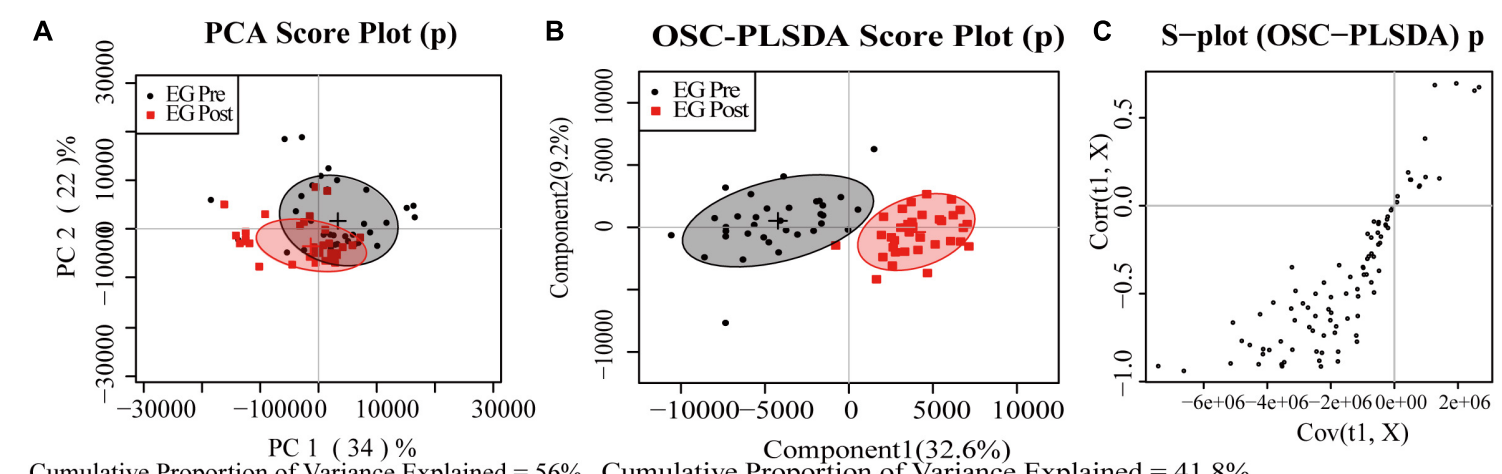

Cumulative Proportion of Variance Explained $=56 \%$ Cumulative Proportion of Variance Explained $=41.8 \%$

D

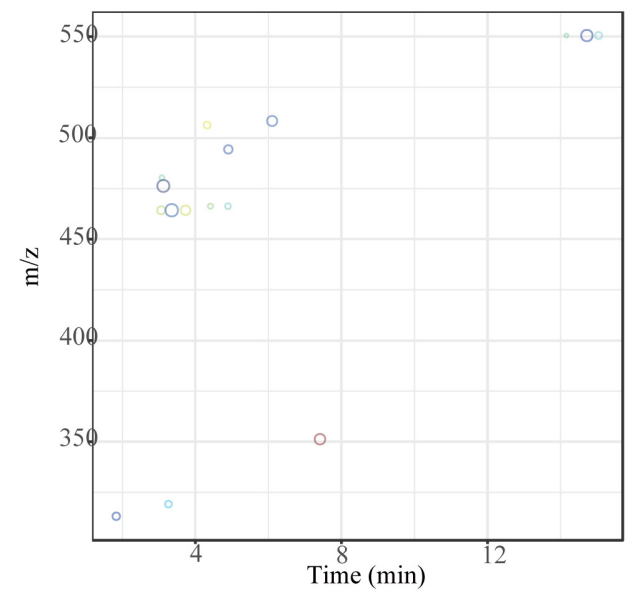

E

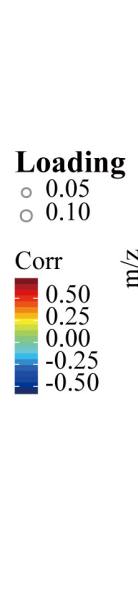

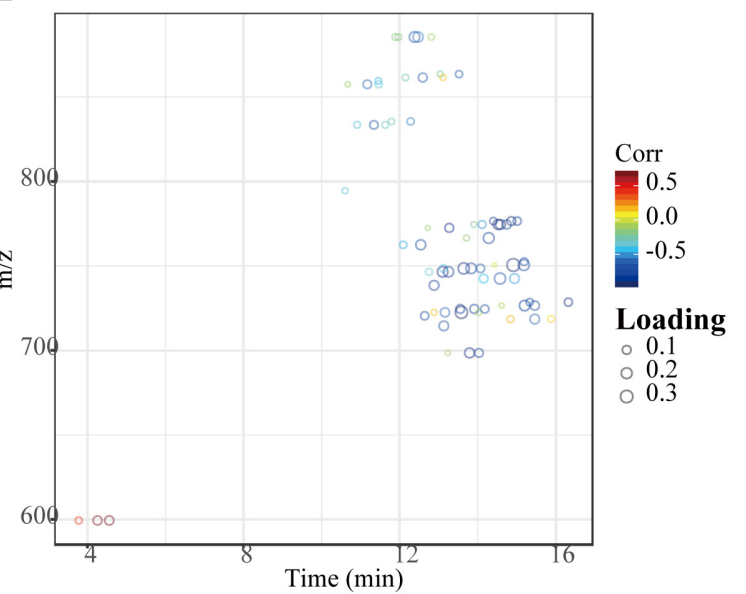

FIGURE 1 | PCA and OPLS-DA analyses of LC-MS for negative ion data from serum of effective patients. (A) PCA Score plot; (B) OPLS-DA Score plot; (C) the corresponding S-plot, points represented differential variables (metabolites): the further away from the center of a variable, the more contribution of the variable to the grouping; (D,E) the color-coded loading plots according to the correlation coefficients, from blue to red, the relativity gradually enhanced. EG, effective group.

adjusted by Benjamini and Hochberg method were calculated (Xu et al., 2017).

\section{Pathway Analysis}

$\mathrm{MetPA}^{2}$ and $\mathrm{KEGG}^{3}$ were used for pathway analysis to identify biologically meaningful metabolic patterns and relevant pathways based on significant differential metabolites. Significant differential metabolites were selected based on OSC-PLS-DA loading/S-plots and FC plots. KEGG enrichment were analyzed in order to detect the functions and pathways of differentially metabolites (Madhu et al., 2018).

\section{RESULTS}

\section{Metabolites Identification}

The results of LC-MS and GC-MS were identified with metabolomics database. LC-MS detected 98 negative icon compounds and 128 positive icon compounds, while GC-MS detected 131 compounds. The results of detection and metabolite

${ }^{2}$ http://www.metaboanalyst.ca

${ }^{3} \mathrm{http}: / /$ www.genome.jp/kegg/ identification for both effective group and ineffective group are listed in Supplementary Tables S1-S3. The combined detection range of the two methods, LC-MS and GCMS, can reflect the level of metabolites in human body comprehensively. Metabolites were identified by querying public metabolomics databases, such as KEGG (see footnote 3) and $\mathrm{HMDB}^{4}$.

\section{Multivariate Analysis}

Results of LC-MS and GC-MS from effective patients $(n=33)$ were taken for a multivariate analysis. For both LC-MS negative ions and positive ions, the PCA score plots exhibited a severe overlap between pre-treatment (Pre) and post-treatment (Post) (Figures 1A, 2A). Applying supervised OSC-PLS-DA eliminated the variations that were unrelated with the grouping discrimination and achieved better separation between Pre and Post groups (Figures 1B, 2B). In the PCA (Figure 3A) and OPLS-DA score plots (Figure 3B) of GC-MS, the Pre and Post groups were satisfactory separated. In the S-plots (Figures 1C, 2C, 3C), points in different shapes represented differential variables (metabolites): the further away from the

\footnotetext{
${ }^{4}$ http://www.hmdb.ca/
} 


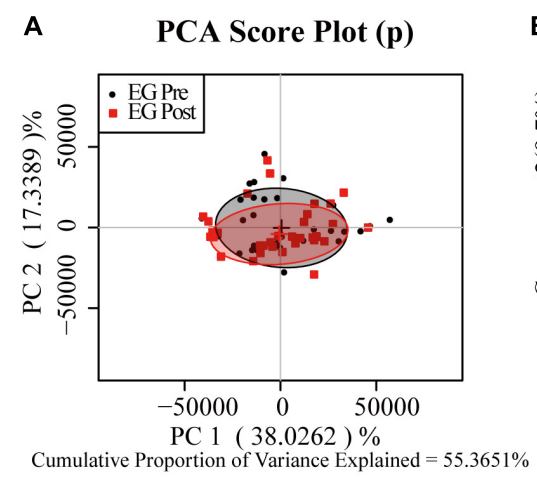

D

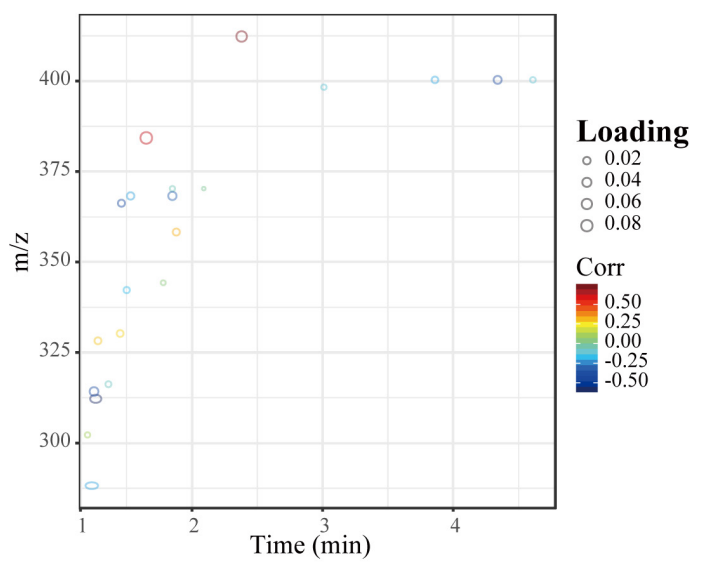

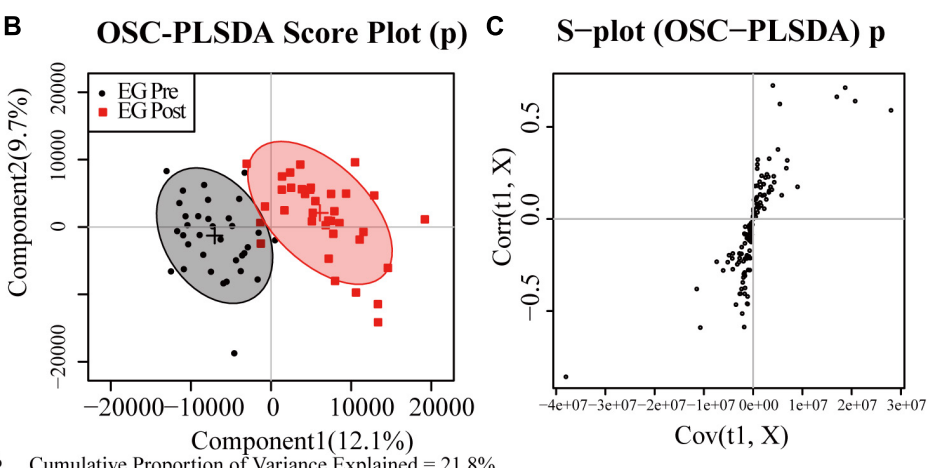

$\mathbf{E}$

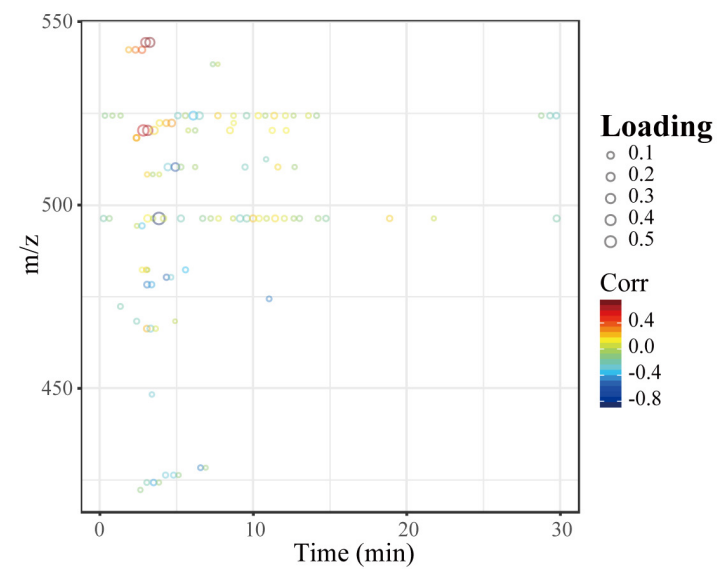

FIGURE 2 | PCA and OPLS-DA analyses of LC-MS for positive ion data from serum of effective patients. (A) PCA Score plot; (B) OPLS-DA Score plot; (C) the corresponding S-plot, points represented differential variables (metabolites): the further away from the center of a variable, the more contribution of the variable to the grouping; (D,E) the color-coded loading plots according to the correlation coefficients, from blue to red, the relativity gradually enhanced. EG, effective group.

center of a variable, the more contribution of the variable to the grouping. The loading plots (Figures 1D,E, 2D,E, 3D,E) were color coded according to the correlation coefficients, from blue to red, the relativity gradually enhanced. They revealed the variation of metabolites in Post group compared with Pre group in serum.

Results of LC-MS and GC-MS from ineffective patients $(n=10)$ were taken for a multivariate analysis using the same method. Supervised OSC-PLS-DA were applied and achieved better separation between Pre and Post groups (Supplementary Figures S1B, S2B, S3B), compared with PCA score plots (Supplementary Figures S1A, S2A, S3A). The color-coded S-plots (Supplementary Figures S1C, S2C, S3C) and loading plots (Supplementary Figures S1D,E, S2D,E, S3D,E) for OPLSDA revealed the variation of metabolites in Post group, compared with Pre group in serum.

\section{Univariate Analysis and Comparison Between Effective Group and Ineffective Group}

Univariate analysis was also carried out to calculate the relative content of each metabolite in Pre group and Post group among effective patients $(n=33)$ and ineffective patients $(n=10)$ by using $\mathrm{R}$ language, and the absolute value of the change rate of each metabolite was obtained. FC was calculated to indicate the degree of variation of differential metabolites.

Among effective patients $(n=33)$, as can be seen in Supplementary Tables S1, S2, LC-MS detected 43 negative ion differential metabolites $(P<0.05)$ and 12 positive ion differential metabolites $(P<0.05)$. In Supplementary Table S3, 108 differential metabolites were detected by GC-MS $(P<0.05)$. Among ineffective patients $(n=10)$, 143 different compounds $(P<0.05)$ were detected by LC-MS and GC-MS. The FC values (Pre vs. Post) of the detected metabolites as well as their associated $P$-values were summarized in Supplementary Tables S1-S3. Red indicates more pre- than post-treatment, meaning the patient's level of metabolite decreased at the end of SCIT treatment. Blue indicates less pre- than post-treatment, meaning the patient's metabolite level rose at the end of this treatment. The darker the red or blue color, the greater the drop or increase.

Through the above results, patients in the effective group and the ineffective group had different metabolic level changes 

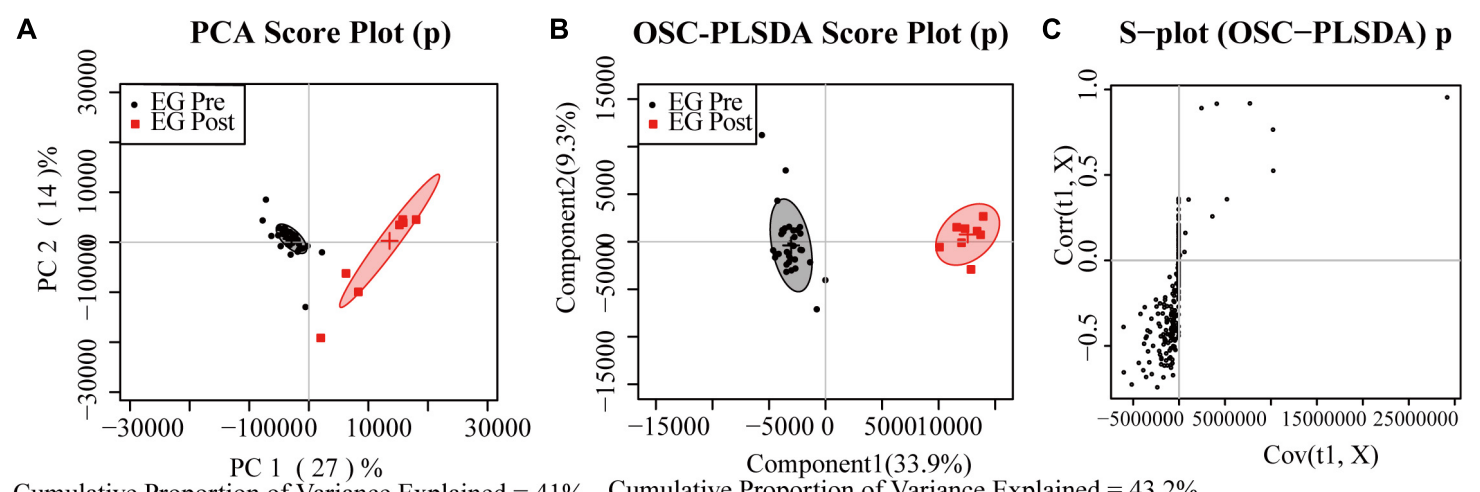

Cumulative Proportion of Variance Explained $=41 \%$ Cumulative Proportion of Variance Explained $=43.2 \%$

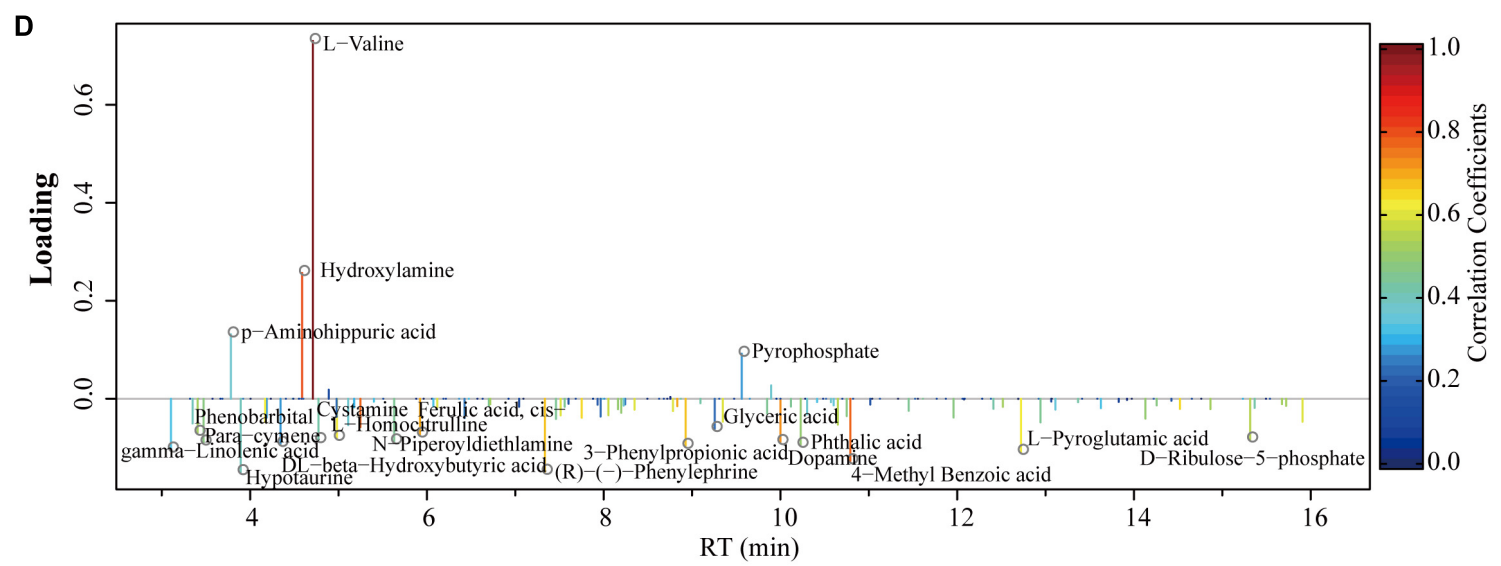

E

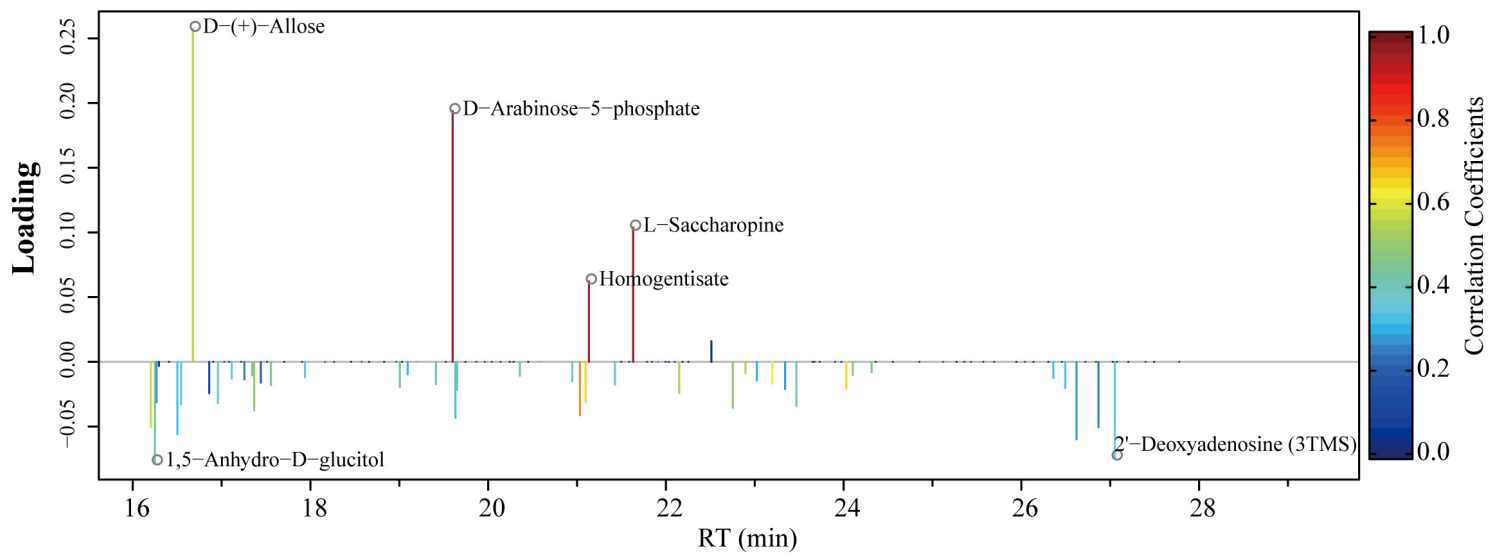

FIGURE 3 | PCA and OPLS-DA analyses of GC-MS data from serum of effective patients. (A) PCA Score plot; (B) OPLS-DA Score plot; (C) the corresponding S-plot, points represented differential variables (metabolites): the further away from the center of a variable, the more contribution of the variable to the grouping; (D,E) the color-coded loading plots according to the correlation coefficients, from blue to red, the relativity gradually enhanced. EG, effective group.

after 1-year SCIT treatment. The results of the ineffective group were taken as negative control, compounds with significant changes in the effective group $(P<0.05)$ but no changes $(P \geq 0.05)$ in the ineffective group or with opposite trends were listed separately in Table 1. There existed 54 compounds changing significantly pre-and-post among effective patients while unchanging among ineffective patients. In particular, metabolism level of L-tyrosine among effective patients was increased after 1-year SCIT treatment, while it was decreased in ineffective patients after treatment.

\section{Metabolite Pathway Analysis of Effective Group}

Based on the above results, significant metabolites in effective group were subjected to pathway analysis using MetPA to explore 
TABLE 1 | Comparison of potential marker metabolites between effective group and ineffective group.

\begin{tabular}{|c|c|c|c|c|c|c|c|}
\hline No. & Compound & EG & IG & No. & Compound & EG & IG \\
\hline 1 & OxFA 18:1+2O; [M-H]- & $\star \star \star$ & & 29 & PE 38:5e; PE 18:1e/20:4; [M-H]- & & \\
\hline 2 & $\mathrm{PI} 36: 4 ; \mathrm{PI} 16: 0-20: 4 ;[\mathrm{M}-\mathrm{H}]-$ & * & & 30 & PE 36:3e; PE 18:1e/18:2; [M-H]- & & \\
\hline 3 & PE 38:6; PE 16:0-22:6; [M-H]- & & & 31 & PE 36:2e; PE 18:0e/18:2; [M-H]- & & \\
\hline 4 & PI 34:1; PI 16:0-18:1; [M-H]- & * & & 32 & PE 36:2e; PE 18:1e/18:1; [M-H]- & & \\
\hline 5 & PI 38:4; PI 18:0-20:4; [M-H]- & * & & 33 & ACar 8:1; [M]+ & & \\
\hline 6 & PE 38:6; PE 16:0-22:6; [M-H]- & & & 34 & ACar 22:6; [M]+ & & \\
\hline 7 & PI 36:2; PI 18:0-18:2; [M-H]- & * & & 35 & ACar 14:2; [M]+ & & \\
\hline 8 & PE 38:7e; PE 16:1e/22:6; [M-H]- & & & 36 & LPE 18:2; [M+H]+ & & \\
\hline 9 & PE 36:4; PE 16:0-20:4; [M-H]- & & & 37 & ACar 20:4; [M]+ & * & \\
\hline 10 & PE 38:7e; PE 16:1e/22:6; [M-H]- & & & 38 & LPC 16:0; [M+H]+ & * & \\
\hline 11 & PE 38:6e; PE 18:2e/20:4; [M-H]- & & & 39 & LPE 18:0; [M+H]+ & & \\
\hline 12 & PE 34:2; PE 16:0-18:2; [M-H]- & & & 40 & ACar 18:0; [M]+ & * & \\
\hline 13 & PE 36:5e; PE 16:1e/20:4; [M-H]- & & & 41 & PC 18:2e; PC 16:2e/2:0; [M+H]+ & * & \\
\hline 14 & PE 38:7e; PE 16:1e/22:6; [M-H]- & & & 42 & PC 20:4e; PC 18:4e/2:0; [M+H]+ & * & \\
\hline 15 & PE 40:8e; PE 18:2e/22:6; [M-H]- & & & 43 & Para-cymene & & * \\
\hline 16 & PE 36:4e; PE 16:0e/20:4; [M-H]- & & & 44 & gamma-Linolenic acid & & \\
\hline 17 & PE 36:5e; PE 16:1e/20:4; [M-H]- & & & 45 & Hypotaurine & & \\
\hline 18 & PE 38:4; PE 18:0-20:4; [M-H]- & & & 46 & L-Valine & $\star$ & \\
\hline 19 & PE 34:3e; PE 16:1e/18:2; [M-H]- & & & 47 & 3-Methyladenine & & \\
\hline 20 & PE 38:6e; PE 18:2e/20:4; [M-H]- & & & 48 & Ferulic acid, cis- & & * \\
\hline 21 & PE 40:7e; PE 18:1e/22:6; [M-H]- & & & 49 & D-Ribulose & & \\
\hline 22 & PE 38:6e; PE 18:1e/20:5; [M-H]- & & & 50 & Succinic semialdehyde & & \\
\hline 23 & PE 36:4e; PE 18:2e/18:2; [M-H]- & & & 51 & 4-Methylbenzoic acid & & \\
\hline 24 & PE 38:4; PE 18:0-20:4; [M-H]- & & & 52 & DL-Threo-b-hydroxyaspartic acid & $\star \star$ & \\
\hline 25 & PE 38:5e; PE 18:1e/20:4; [M-H]- & & & 53 & 5-Aminovaleric acid & & \\
\hline 26 & PE 40:7e; PE 18:1e/22:6; [M-H]- & & & 54 & L-Pyroglutamic acid & & \\
\hline 27 & PE 36:2; PE 18:0-18:2; [M-H]- & & & 55 & L-Tyrosine & ** & * \\
\hline 28 & PE 40:7e; PE 18:1e/22:6; [M-H]- & & & & & & \\
\hline
\end{tabular}

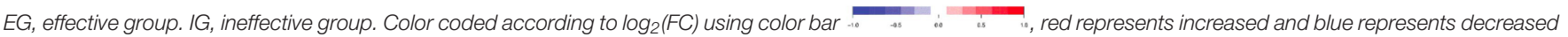

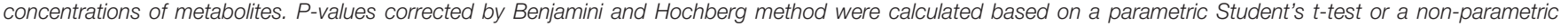

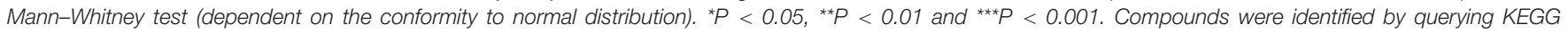
(http://www.genome.jp/kegg/) and HMDB (http://www.hmdb.ca/).

the most impacted pathways in patients who received 1-year SCIT and achieved effective result.

Bubble plot (Figure 4) shown the related metabolic pathways in effective group. There are 11 main metabolic pathways $(P<0.05)$ involved, shown in Table 2 with their related compounds. For pathway enrichment analysis we used the databases provided by KEGG, identified 30 compounds that were significantly enriched $(P<0.05)$, spanning 22 pathways (Figure 5). Red circles refer to KEGG pathways, related modules, enzymes, reactions and compounds were also analyzed.

\section{DISCUSSION}

Allergic rhinitis caused by pollen is usually seasonal allergic rhinitis with obvious seasonal characteristics. Allergic symptoms occur in the pollen season, but no symptoms appear in the non-pollen seasons, which is thought to be related to pollen exposure and pollen concentration in the air (Wakamiya et al., 2019; Xie et al., 2019). SIT is considered as the only way to eradicate for allergic rhinitis. However, its clinical application still has some shortcomings: there are no convincing objective indicators to evaluate the clinical efficacy; the mechanism of action is too complex and unclear. Metabolites reflect the changes of cell function after disease or external stimulation, especially the related enzymatic or chemical reactions (Schrimpe-Rutledge et al., 2016; Zampieri et al., 2017). Therefore, metabolomics is widely used in complex diseases, such as cancer, cardiovascular disease and diabetes mellitus. It is often used to diagnose diseases, understand disease mechanisms, identify new drug targets, customize drug treatment and monitor therapeutic effects (Wishart, 2016; Newgard, 2017). Allergic diseases are also complex and heterogeneous diseases suitable for metabolomics research (Bunyavanich and Schadt, 2015). Metabolomics has been extensively studied on the effects of metabolism on the progression of allergic diseases, especially asthma (Kelly et al., 2017; Reinke et al., 2017). It has the potential of diagnosis and treatment, and is considered to be an effective method to find biomarkers of allergic diseases (Villaseñor et al., 2017). Up to now, the research on the therapeutic methods of allergic rhinitis based on metabonomics is limited. There 


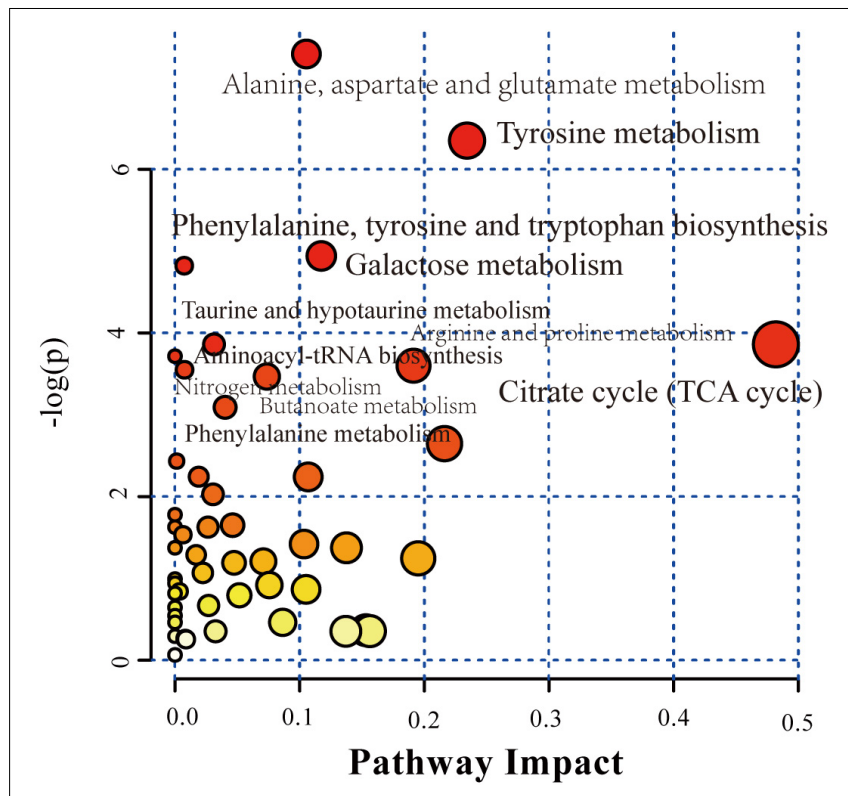

FIGURE 4 | Pathway analysis of significant metabolites in serum of effective patients between Pre and Post groups. Bubble plot of the altered metabolic pathways in the serum of pre group compared with post group. Bubble area is proportional to the impact of each pathway, with color denoting the significance from highest in red to lowest in white. The labels in the figures correspond to KEGG ID.

were two animal level studies that have found the metabolic mechanism of drug treatment for allergic rhinitis (Zhuang et al., 2018; Chen et al., 2019). However, there is a blank in the metabolomics study of SCIT.

In our study, we recruited 78 allergic rhinitis patients with Artemisia sieversiana pollen allergy during the pollen season, and finally 43 were included in the analysis. They were all treated with SCIT for 1 year. In addition to the detailed inclusion criteria in the method above, other covariates, such as genders and fasting or not, were not taken into account, since the current study on human does not indicate the impact of other covariates on the results (Adamko et al., 2018; Ma et al., 2019). Through metabolomics detection and analysis of serum from these patients before and after treatment, we studied the difference of metabolic levels in Artemisia sieversiana pollen allergic rhinitis patients before and after 1-year of SCIT treatment by metabonomic method, and combined with the physiological and biochemical significance of different metabolites and related metabolic pathways, we made some positive discoveries.

Firstly, SCIT currently has no objective clinical evaluation indicators or biomarkers. Only subjective indicators such as therapeutic index can be used for the evaluation of curative effect, which makes the clinical evaluation of this therapy lack scientific to a certain extent. Therefore, in our study, by using ineffective patients as negative control, we found metabolites that changed significantly in the effective patients but remained unchanged or opposite in the ineffective patients. Among them, L-tyrosine was increased in the effective patients and decreased in the ineffective patients, which is an opposite trend (Table 1).
TABLE 2 | Pathway analysis and related significant metabolites of effective group.

\begin{tabular}{|c|c|c|c|c|}
\hline No. & Pathways & Compounds & KEGG ID & $\mathbf{P}$ \\
\hline \multirow[t]{5}{*}{1} & \multirow{5}{*}{$\begin{array}{l}\text { Alanine, } \\
\text { aspartate and } \\
\text { glutamate } \\
\text { metabolism }\end{array}$} & L-Asparagine & C00152 & 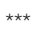 \\
\hline & & Fumaric acid & $\mathrm{C} 00122$ & \\
\hline & & N-Carbamoyl-L-aspartate & $\mathrm{C} 00438$ & \\
\hline & & Succinic semialdehyde & C00232 & \\
\hline & & Succinic acid & $\mathrm{C} 00042$ & \\
\hline \multirow[t]{6}{*}{2} & \multirow{6}{*}{$\begin{array}{l}\text { Tyrosine } \\
\text { metabolism }\end{array}$} & L-Tyrosine & C00082 & ** \\
\hline & & 4-Hydroxyphenethyl alcohol & C06044 & \\
\hline & & 3,4-Dihydroxy-L-phenylalanine & C00355 & \\
\hline & & Homogentisate & C00544 & \\
\hline & & Dopamine & C03758 & \\
\hline & & Fumaric acid & C00122 & \\
\hline \multirow[t]{5}{*}{3} & \multirow{5}{*}{$\begin{array}{l}\text { Galactose } \\
\text { metabolism }\end{array}$} & Glycerol & $\mathrm{C} 00116$ & ** \\
\hline & & D-(-)-Sorbitol & C00794 & \\
\hline & & Alpha-Lactose & $\mathrm{C} 00243$ & \\
\hline & & Galactitol & C01697 & \\
\hline & & Myo-Inositol & $\mathrm{C} 00137$ & \\
\hline \multirow[t]{4}{*}{4} & \multirow{4}{*}{$\begin{array}{l}\text { Phenylalanine, } \\
\text { tyrosine and } \\
\text { tryptophan } \\
\text { biosynthesis }\end{array}$} & Phosphoenolpyruvic acid & C00074 & ** \\
\hline & & 3,4-Dihydroxybenzoic acid & $\mathrm{C} 00230$ & \\
\hline & & L-Tyrosine & C00082 & \\
\hline & & L-Tryptophane & $\mathrm{C} 00078$ & \\
\hline \multirow[t]{3}{*}{5} & \multirow{3}{*}{$\begin{array}{l}\text { Citrate cycle } \\
\text { (TCA cycle) }\end{array}$} & Succinic acid & C00042 & * \\
\hline & & Fumaric acid & C00122 & \\
\hline & & Phosphoenolpyruvic acid & $\mathrm{C} 00074$ & \\
\hline \multirow[t]{3}{*}{6} & \multirow{3}{*}{$\begin{array}{l}\text { Taurine and } \\
\text { hypotaurine } \\
\text { metabolism }\end{array}$} & L-Cysteic acid & C00506 & * \\
\hline & & Hypotaurine & C00519 & \\
\hline & & Taurine & C00245 & \\
\hline \multirow[t]{6}{*}{7} & \multirow{6}{*}{$\begin{array}{l}\text { Aminoacyl- } \\
\text { tRNA } \\
\text { biosynthesis }\end{array}$} & L-Asparagine & C00152 & * \\
\hline & & L-Valine & $\mathrm{C} 00183$ & \\
\hline & & L-Leucine & C00123 & \\
\hline & & L-Tryptophane & C00078 & \\
\hline & & L-Tyrosine & C00082 & \\
\hline & & L-Proline & C00148 & \\
\hline \multirow[t]{6}{*}{8} & \multirow{6}{*}{$\begin{array}{l}\text { Arginine and } \\
\text { proline } \\
\text { metabolism }\end{array}$} & Fumaric acid & $\mathrm{C} 00122$ & * \\
\hline & & L-Proline & C00148 & \\
\hline & & Urea & C00086 & \\
\hline & & Putrescine & C00134 & \\
\hline & & Creatinine & C00791 & \\
\hline & & 5-Aminovaleric acid & $\mathrm{C} 00431$ & \\
\hline \multirow[t]{4}{*}{9} & \multirow{4}{*}{$\begin{array}{l}\text { Nitrogen } \\
\text { metabolism }\end{array}$} & L-Tyrosine & C00082 & * \\
\hline & & L-Tryptophane & C00078 & \\
\hline & & Taurine & $\mathrm{C} 00245$ & \\
\hline & & L-Asparagine & $\mathrm{C} 00152$ & \\
\hline \multirow[t]{4}{*}{10} & \multirow{4}{*}{$\begin{array}{l}\text { Butanoate } \\
\text { metabolism }\end{array}$} & Succinic semialdehyde & C00232 & * \\
\hline & & Succinic acid & C00042 & \\
\hline & & Fumaric acid & $\mathrm{C} 00122$ & \\
\hline & & DL-beta-Hydroxybutyric acid & C01089 & \\
\hline \multirow[t]{4}{*}{11} & Phenylalanine & L-Tyrosine & C00082 & * \\
\hline & & Fumaric acid & C00122 & \\
\hline & & Succinic acid & C00042 & \\
\hline & & 3-Phenylpropionic acid & C05629 & \\
\hline
\end{tabular}

P-values corrected by Benjamini and Hochberg method were calculated based on a parametric Student's t-test or a non-parametric Mann-Whitney test (dependent on the conformity to normal distribution). ${ }^{*} P<0.05$, ${ }^{* *} P<0.01$ and ${ }^{* * *} P<0.001$. 


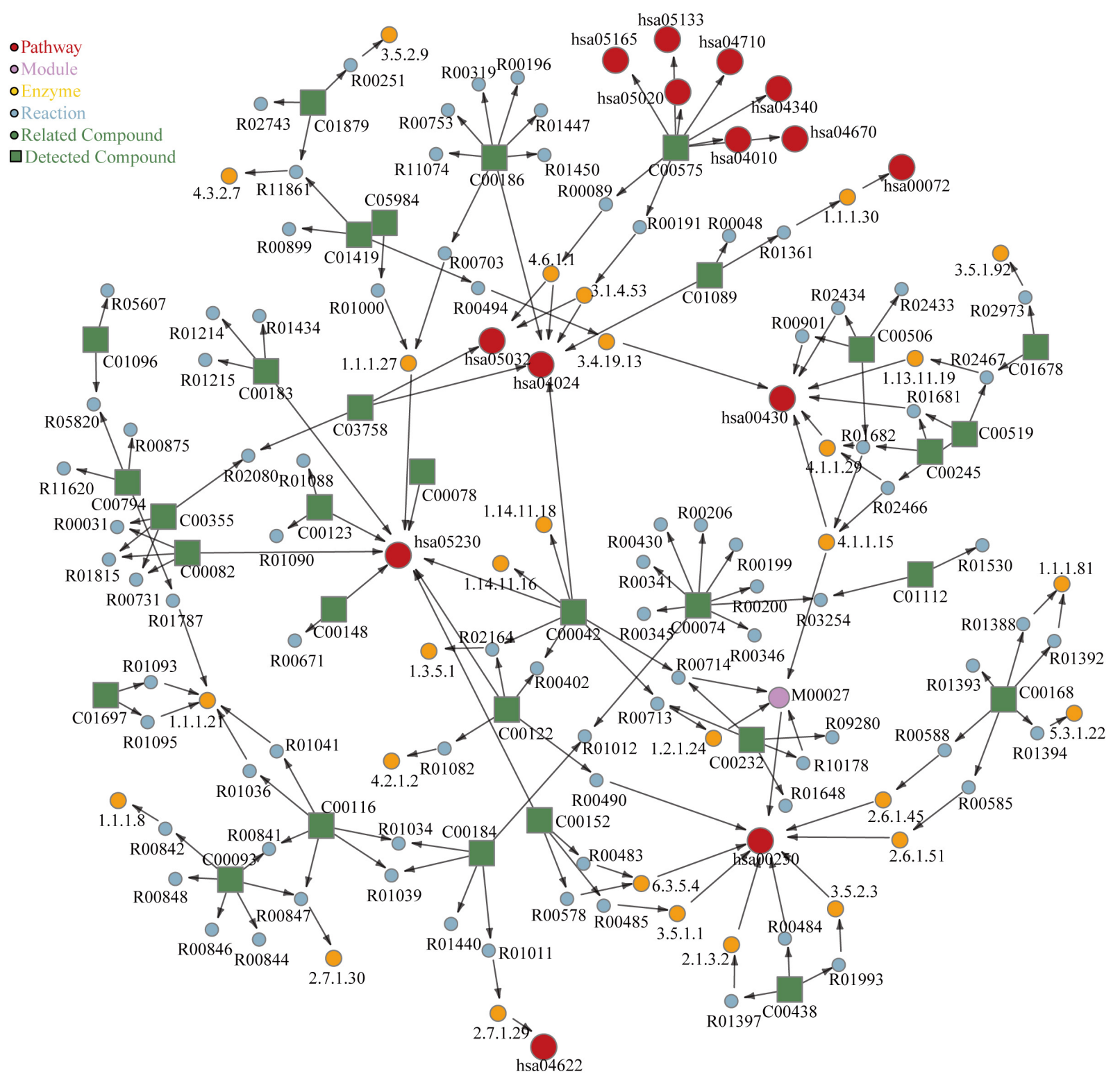

FIGURE 5 | KEGG enrichment of metabolites in serum of effective patients between Pre and Post groups. The labels in the figure corresponds to KEGG ID.

At the same time, this compound was involved in five metabolic pathways related in effective patients (Table 2). L-Tyrosine is one of the ingredients of MPL adjuvant of allergy vaccine, which assists in redressing a healthy balance between TH1- and TH2type activities and enhances production of allergen-specific IgG (Patel et al., 2014). Combined with our findings, we can infer that L-tyrosine metabolism in patients may affect the treatment effect, and be a key factor that leads to effective or ineffective treatment result. These phenomena showed that L-tyrosine could be used as a biomarker to evaluate clinical efficacy of SCIT.

On the other hand, through metabolomics analysis of patients who were effective in 1-year SCIT treatment, we found that the levels of various metabolites in the body changed significantly after patients received SCIT (Supplementary Tables S1-S3), involving 11 metabolic pathways (Table 2). These changes are likely to reveal the impact of SCIT treatment on patients and may be related to the mechanism of SCIT.

This study found that there were significant disorders in nitric oxide (NO) related metabolism before and after treatment, including arginine and proline metabolism, tyrosine metabolism, and nitrogen metabolism. NO acts as an inflammatory mediator in the airway (Kim et al., 2016). NO imbalance is considered to play an important role in the pathogenesis of allergic rhinitis, and nasal $\mathrm{NO}$ has been proved to be the best biomarker for distinguishing AR from non-AR (Takeno et al., 2014). $\mathrm{NO}$ is synthesized from L-arginine by nitric oxide synthase, which also plays an important role in the pathophysiology of rhinitis, especially in the glandular function of allergic nasal 
mucosa (Kang et al., 2000). Nitric oxide synthase is expressed in many cell types, such as epithelial cells, inflammatory cells (macrophages, neutrophils, and mast cells), airway nerves and vascular endothelial cells (Kim et al., 2016). The recruitment of eosinophils is accompanied by an increase in $\mathrm{NO}$ in the nasal cavity, which leads to oxidative stress (Hanazawa et al., 2000). Eosinophils are considered to be responsible for allergicrelated inflammatory diseases. Peripheral blood eosinophils are biomarkers of allergic airway inflammation (Amin et al., 2016). Studies have found that fractional exhaled $\mathrm{NO}$ and blood eosinophil levels are significantly correlated with index\%Tfh2 cells per\% Breg cells (Kamekura et al., 2015). High-dose allergen administered resulting in immune deviation from a Th2 to a Th1driven response is considered to be one of the mechanisms of immunological and clinical tolerance in SIT (Jasper et al., 2017). In perennial allergic rhinitis, inducible nitric oxide synthase in nasal mucosa is up-regulated, and NO in nasal cavity decreases after treatment with topical corticosteroids (Hanazawa et al., 2000). These evidences suggest that SCIT treatment of rhinitis may play a role in the production and activity of NO, thereby improving the symptoms and diseases of patients with rhinitis.

In addition, arginine is a substrate for nitric oxide synthase and arginase. Arginase expression is strongly induced by cytokines, in particular IL-4 and IL-13, which are produced at elevated level in airways and which activate inflammatory pathways. Arginase provides a precursor for polyamines and proline by modulating nitric oxide synthase activity, which stimulate cell growth and collagen synthesis (Lewandowicz and Pawliczak, 2007). Arginase competes with nitric oxide synthase for L-arginine, which catalyzes the hydrolysis of arginine to urea and ornithine (Yasar et al., 2011). Therefore, an increase in serum arginase activity may limit the formation of NO catalyzed by inducible nitric oxide synthase, resulting in allergic rhinitis (Maarsingh et al., 2006). The metabolic disorders of arginine and proline metabolism in this study may be closely related to the effective treatment of SCIT.

In our study, taurine and taurine metabolic levels increased significantly in patients with allergic rhinitis after 1 year of treatment. Taurine and taurine metabolism were also found to increase after asthma treatment (Comhair et al., 2015; QuanJun et al., 2017), which may be related to taurine inhibiting the production of inflammatory cytokines, reducing nasal friction and histamine (Nam et al., 2017). TCA cycle (Quan-Jun et al., 2017; Khoo et al., 2018; Chen et al., 2019) and phenylalanine, tyrosine, and tryptophan biosynthesis (Zinkeviciene et al., 2016) has also been found to be of some significance in the study of metabolomics of many allergies. These findings have certain indications and auxiliary effects for researchers to further discover the mechanism of SCIT for allergic rhinitis.

\section{CONCLUSION}

Our metabolomics studies have found some differential metabolites associated with the SCIT treatment of Artemisia sieversiana pollen allergic rhinitis. We found that L-tyrosine could be used as a biomarker to evaluate clinical efficacy of SCIT, because of its opposite trend in effective patients and ineffective patients. At the same time, the metabolic pathways related to NO production and metabolism in vivo were obviously disordered after 1 year of treatment for allergic rhinitis. We can infer that the mechanism of immunotherapy may be closely related to $\mathrm{NO}$ and nitric oxide synthase. However, further replication studies are necessary to validate our inference. These studies may provide a new perspective with which to find potential biomarkers of and understand the mechanism of SCIT, as well as a new reference for personalized treatment.

\section{DATA AVAILABILITY STATEMENT}

The data used to support the findings of this study are available from the corresponding author upon reasonable request.

\section{ETHICS STATEMENT}

The studies involving human participants were reviewed and approved by the Institutional Review Board of Beijing Shijitan Hospital, Affiliated to Capital Medical University. Written informed consent to participate in this study was provided by the participants' legal guardian/next of kin.

\section{AUTHOR CONTRIBUTIONS}

H-YS, T-TM, Y-LC, W-JY, and J-GL completed the work of sample collection and patient information collection. CP completed the experiment and data analysis and wrote this thesis. M-DC helped to data analysis. J-FW, X-YW, D-YW, and $\mathrm{H}-\mathrm{DH}$ conceived of the study and participated in its design and coordination and helped to draft the manuscript. All authors read and approved the final manuscript.

\section{FUNDING}

This research was partially supported by Beijing Municipal Administration of Hospitals Clinical Medicine Development of Special Funding Support (code: ZYLX201826) and Beijing Municipal Science \& Technology Commission (code: Z161100000516006).

\section{ACKNOWLEDGMENTS}

We thank all patients for their participation in the study. We also thank Professor Jun-Song Wang (Nanjing University of Science and Technology, Nanjing, China) for technical advice.

\section{SUPPLEMENTARY MATERIAL}

The Supplementary Material for this article can be found online at: https://www.frontiersin.org/articles/10.3389/fphar.2020. 00305/full\#supplementary-material 


\section{REFERENCES}

Adamko, D. J., Khamis, M. M., Steacy, L. M., Regush, S., Bryce, R., and Ellis, A. K. (2018). Severity of allergic rhinitis assessed by using urine metabolomic profiling: proof of concept. J. Allergy Clin. Immunol. 142, 687.e6-689.e6. doi: 10.1016/j.jaci.2018.02.051

Amin, K., Janson, C., and Bystrom, J. (2016). Role of eosinophil granulocytes in allergic airway inflammation endotypes. Scand. J. Immunol. 84, 75-85. doi: $10.1111 /$ sji. 12448

Bachmann, M. F., and Kündig, T. M. (2017). Allergen-specific immunotherapy: is it vaccination against toxins after all? Allergy 72, 13-23. doi: 10.1111/all.12890

Brożek, J. L., Bousquet, J., Agache, I., Agarwal, A., Bachert, C., Bosnic-Anticevich, S., et al. (2017). Allergic Rhinitis and its Impact on Asthma (ARIA) guidelines2016 revision. J. Allergy Clin. Immunol. 140, 950-958. doi: 10.1016/j.jaci.2017. 03.050

Bunyavanich, S., and Schadt, E. E. (2015). Systems biology of asthma and allergic diseases: a multiscale approach. J. Allergy Clin. Immunol. 135, 31-42. doi: 10. 1016/j.jaci.2014.10.015

Chen, S., Chen, G., Shu, S., Xu, Y., and Ma, X. (2019). Metabolomics analysis of baicalin on ovalbumin-sensitized allergic rhinitis rats. R. Soc. Open Sci. 6:181081. doi: 10.1098/rsos.181081

Chen, T., Sheng, J., Fu, Y., Li, M., Wang, J., and Jia, A.-Q. (2017). 1H NMRbased global metabolic studies of Pseudomonas aeruginosa upon exposure of the quorum sensing inhibitor resveratrol. J. Proteome Res. 16, 824-830. doi: 10.1021/acs.jproteome.6b00800

Comhair, S. A., McDunn, J., Bennett, C., Fettig, J., Erzurum, S. C., and Kalhan, S. C. (2015). Metabolomic endotype of asthma. J. Immunol. 195, 643-650. doi: 10.4049/jimmunol.1500736

Cox, L., Nelson, H., Lockey, R., Calabria, C., Chacko, T., Finegold, I., et al. (2011). Allergen immunotherapy: a practice parameter third update. J. Allergy Clin. Immunol. 127(1 Suppl.), S1-S55. doi: 10.1016/j.jaci.2010.09.034

Cui, L., and Yin, J. (2018). Association of serum specific IgE levels with asthma in autumn pollen-induced allergic rhinitis: a retrospective analysis. J. Asthma 56, 505-511. doi: 10.1080/02770903.2018.1466316

Gao, Z., Fu, W. Y., Sun, Y., Gao, B., Wang, H. Y., Liu, M., et al. (2019). Artemisia pollen allergy in China: component-resolved diagnosis reveals allergic asthma patients have significant multiple allergen sensitization. Allergy 74, 284-293. doi: $10.1111 /$ all.13597

Greenhawt, M., Oppenheimer, J., Nelson, M., Nelson, H., Lockey, R., Lieberman, P., et al. (2017). Sublingual immunotherapy: a focused allergen immunotherapy practice parameter update. Ann. Allergy Asthma Immunol. 118, 276.e2-282.e2. doi: 10.1016/j.anai.2016.12.009

Greiner, A. N., Hellings, P. W., Rotiroti, G., and Scadding, G. K. (2011). Allergic rhinitis. Lancet 378, 2112-2122. doi: 10.1016/s0140-6736(11)60130-X

Gu, Z. Y., and Dong, Z. (2005). Principles and recommendations for diagnosis and treatment of allergic rhinitis. Chin. J. Otorhinolaryngol. Head Neck Surg. 40, 166-167.

Hanazawa, T., Antuni, J. D., Kharitonov, S. A., and Barnes, P. J. (2000). Intranasal administration of eotaxin increases nasal eosinophils and nitric oxide in patients with allergic rhinitis. J. Allergy Clin. Immunol. 105, 58-64. doi: 10.1016/ s0091-6749(00)90178-7

Hong, W., Liu, Y., Li, M.-H., Xing, Y.-X., Chen, T., Fu, Y.-H., et al. (2018). In vivo toxicology of carbon dots by $1 \mathrm{H}$ NMR-based metabolomics. Toxicol. Res. 7, 834-847. doi: 10.1039/c8tx00049b

Hu, S. J., Wei, P., Kou, W., Wu, X. F., Liu, M. Y., Chen, C., et al. (2017). Prevalence and risk factors of allergic rhinitis:a meta-analysis. J. Clin. Otorhinolaryngol. Head Neck Surg. 31, 1485-1491. doi: 10.13201/j.issn.1001-1781.2017. 19.006

Hu, W. H., Zhu, L., Xie, L. F., Zhang, F. Z., Bai, M. Y., Wang, N., et al. (2017). Correlation between the visiting rate of patients with allergic rhinitis and airborne pollen concentrations in Beijing in recent 3 years. Clin. J. Otorhinolaryngol. Head Neck Surg. 52, 31-36. doi: 10.3760/cma.j.isn.1673-0860. 2017.01.006

Jacobsen, L., Niggemann, B., Dreborg, S., Ferdousi, H. A., Halken, S., Host, A., et al. (2007). Specific immunotherapy has long-term preventive effect of seasonal and perennial asthma: 10-year follow-up on the PAT study. Allergy 62, 943-948. doi: $10.1111 / \mathrm{j} .1398-9995.2007 .01451 . x$
Jasper, H. K., Stephen, R. D., Hans, I. T. V., and Mohamed, H. S. (2017). Applications and mechanisms of immunotherapy in allergic rhinitis and asthma. Ther. Adv. Respir. Dis. 11, 73-86. doi: 10.1177/1753465816669662

Johnson, C. H., Spilker, M. E., Goetz, L., Peterson, S. N., and Siuzdak, G. (2016). Metabolite and microbiome interplay in cancer immunotherapy. Cancer Res. 76, 6146-6152. doi: 10.1158/0008-5472.Can-16-0309

Kamekura, R., Shigehara, K., Miyajima, S., Jitsukawa, S., Kawata, K., Yamashita, K., et al. (2015). Alteration of circulating type 2 follicular helper $\mathrm{T}$ cells and regulatory $\mathrm{B}$ cells underlies the comorbid association of allergic rhinitis with bronchial asthma. Clin. Immunol. 158, 204-211. doi: 10.1016/j.clim.2015. 02.016

Kang, B.-H., Chen, S.-S., Jou, L.-S., Weng, P.-K., and Wang, H.-W. (2000). Immunolocalization of inducible nitric oxide synthase and 3-nitrotyrosine in the nasal mucosa of patients with rhinitis. Eur. Arch. Otorhinolaryngol. 257, 242-246. doi: 10.1007/s004050050231

Kelly, R. S., Virkud, Y., Giorgio, R., Celedón, J. C., Weiss, S. T., and Lasky-Su, J. (2017). Metabolomic profiling of lung function in Costa-Rican children with asthma. Biochim. Biophys. Acta Mol. Basis Dis. 1863, 1590-1595. doi: 10.1016/j. bbadis.2017.02.006

Khoo, L. W., Audrey Kow, S. F., Maulidiani, M., Lee, M. T., Tan, C. P., Shaari, K., et al. (2018). Plasma and urine metabolite profiling reveals the protective effect of Clinacanthus nutans in an ovalbumin-induced anaphylaxis model: (1)H-NMR metabolomics approach. J. Pharm. Biomed. Anal. 158, 438-450. doi: 10.1016/j.jpba.2018.06.038

Kim, H.-B., Eckel, S. P., Kim, J. H., and Gilliland, F. D. (2016). Exhaled NO: determinants and clinical application in children with allergic airway disease. Allergy Asthma Immunol. Res. 8, 12-21. doi: 10.4168/aair.2016.8.1.12

Lewandowicz, A. M., and Pawliczak, R. (2007). Arginine metabolism in bronchial asthma. Postepy. Hig. Med. Dosw. 61, 156-166.

Li, M.-H., Du, H.-Z., Kong, G.-J., Liu, L.-B., Li, X.-X., Lin, S.-S., et al. (2017). Nuclear magnetic resonance-based metabolomics approach to evaluate the prevention effect of Camellia nitidissima chi on colitis-associated carcinogenesis. Front. Pharmacol. 8:447. doi: 10.3389/fphar.2017.00447

Lv, Y., Wang, J., Xu, D., Liao, S., Li, P., Zhang, Q., et al. (2017). Comparative study of single/combination use of Huang-Lian-Jie-Du decoction and berberine on their protection on sepsis induced acute liver injury by NMR metabolic profiling. J. Pharm. Biomed. Anal. 145, 794-804. doi: 10.1016/j.jpba.2017.07.062

Ma, G. C., Wang, T. S., Wang, J., Ma, Z. J., and Pu, S. B. (2019). Serum metabolomics study of patients with allergic rhinitis. Biomed. Chromatogr. 34:e4739. doi: 10.1002/bmc.4739

Ma, R., Shen, Y., Hou, L., Yang, Z., Feng, N., Yan, X., et al. (2014). The correlation of allergic rhinitis and trace elements in Ningxia region. J. Clin. Otorhinolaryngol. Head Neck Surg. 49, 1017-1020.

Maarsingh, H., Leusink, J., Zaagsma, J., and Meurs, H. (2006). Role of the L-citrulline/L-arginine cycle in iNANC nerve-mediated nitric oxide production and airway smooth muscle relaxation in allergic asthma. Eur. J. Pharmacol. 546, 171-176. doi: 10.1016/j.ejphar.2006.07.041

Madhu, L. N., Upadhya, R., Kodali, M., and Shetty, A. K. (2018). Emerging antiaging strategies - scientific basis and efficacy. Aging Dis. 9, 1165-1184. doi: 10.14336/ad.2018.1026

Nam, S.-Y., Kim, H.-M., and Jeong, H.-J. (2017). The potential protective role of taurine against experimental allergic inflammation. Life Sci. 184, 18-24. doi: 10.1016/j.lfs.2017.07.007

Newgard, C. B. (2017). Metabolomics and metabolic diseases: where do we stand? Cell Metab. 25, 43-56. doi: 10.1016/j.cmet.2016.09.018

Noon, L. (1911). Prophylactic inoculation against hay fever. Lancet 177, 15721573. doi: 10.1016/s0140-6736(00)78276-6

Okubo, K., Kurono, Y., Ichimura, K., Enomoto, T., Okamoto, Y., Kawauchi, H., et al. (2017). Japanese guidelines for allergic rhinitis 2017. Allergol. Int. 66, 205-219. doi: 10.1016/j.alit.2016.11.001

Omabe, M., Elom, S., and Omabe, K. N. (2018). Emerging metabolomics biomarkers of polycystic ovarian syndrome; targeting the master metabolic disrupters for diagnosis and treatment. Endocr. Metab. Immune Disord. Drug Targets 18, 221-229. doi: 10.2174/1871530318666180122165415

Passalacqua, G., Canonica, G. W., and Bagnasco, D. (2016). Benefit of SLIT and SCIT for allergic rhinitis and asthma. Curr. Allergy Asthma Rep. 16:88. doi: 10.1007/s11882-016-0666-x 
Patel, P., Holdich, T., Fischer von Weikersthal-Drachenberg, K. J., and Huber, B. (2014). Efficacy of a short course of specific immunotherapy in patients with allergic rhinoconjunctivitis to ragweed pollen. J. Allergy Clin. Immunol. 133, 121.e2-129.e2. doi: 10.1016/j.jaci.2013.05.032

Polosa, R., Li Gotti, F., Mangano, G., Paolino, G., Mastruzzo, C., Vancheri, C., et al. (2004). Effect of immunotherapy on asthma progression, BHR and sputum eosinophils in allergic rhinitis. Allergy 59, 1224-1228. doi: 10.1111/j.1398-9995. 2004.00537.x

Quan-Jun, Y., Jian-Ping, Z., Jian-Hua, Z., Yong-Long, H., Bo, X., Jing-Xian, Z., et al. (2017). Distinct metabolic profile of inhaled budesonide and salbutamol in asthmatic children during acute exacerbation. Basic Clin. Pharmacol. Toxicol. 120, 303-311. doi: 10.1111/bcpt.12686

Reinke, S. N., Gallart-Ayala, H., Gómez, C., Checa, A., Fauland, A., Naz, S., et al. (2017). Metabolomics analysis identifies different metabotypes of asthma severity. Eur. Respir. J. 49:1601740. doi: 10.1183/13993003.01740-2016

Schrimpe-Rutledge, A. C., Codreanu, S. G., Sherrod, S. D., and McLean, J. A. (2016). Untargeted metabolomics strategies-challenges and emerging directions. J. Am. Soc. Mass Spectrom. 27, 1897-1905. doi: 10.1007/s13361-0161469-y

Song, Y., Wang, M., Xie, J., Li, W., Zhang, X., Wang, T., et al. (2015). Prevalence of allergic rhinitis among elementary and middle school students in Changsha city and its impact on quality of life. J. Laryngol. Otol. 129, 1108-1114. doi: $10.1017 /$ S0022215115002492

Takeno, S., Yoshimura, H., Kubota, K., Taruya, T., Ishino, T., and Hirakawa, K. (2014). Comparison of nasal nitric oxide levels between the inferior turbinate surface the middle meatus in patients with symptomatic allergic rhinitis. Allergol. Int. 63, 475-483. doi: 10.2332/allergolint.14-OA-0689

Tang, R., Sun, J.-L., Yin, J., and Li, Z. (2015). Artemisia allergy research in China. Biomed. Res. Int. 2015:179426. doi: 10.1155/2015/179426

Villaseñor, A., Rosace, D., Obeso, D., Pérez-Gordo, M., Chivato, T., Barbas, C., et al. (2017). Allergic asthma: an overview of metabolomic strategies leading to the identification of biomarkers in the field. Clin. Exp. Allergy 47, 442-456. doi: 10.1111/cea.12902

Wakamiya, S., Matsune, S., Okubo, K., and Aramaki, E. (2019). Causal relationships among pollen counts, tweet numbers, and patient numbers for seasonal allergic rhinitis surveillance: retrospective analysis. J. Med. Internet Res. 21:e10450. doi: 10.2196/10450

Wallace, D. V., and Dykewicz, M. S. (2017). Comparing the evidence in allergic rhinitis guidelines. Curr. Opin. Allergy Clin. Immunol. 17, 286-294. doi: 10. 1097/ACI.0000000000000375

Wan, C., Li, P., Chen, C., Peng, X., Li, M., Chen, M., et al. (2017). Antifungal activity of ramulus cinnamomi explored by $1 \mathrm{H}-\mathrm{NMR}$ based metabolomics approach. Molecules 22:2237. doi: 10.3390/molecules22122237

Wang, X. D., Zheng, M., Lou, H. F., Wang, C. S., Zhang, Y., Bo, M. Y., et al. (2016). An increased prevalence of self-reported allergic rhinitis in major Chinese cities from 2005 to 2011. Allergy 71, 1170-1180. doi: 10.1111/all.12874

Wang, X. Y., Ma, T. T., Wang, X. Y., Zhuang, Y., Wang, X. D., Ning, H. Y., et al. (2018). Prevalence of pollen-induced allergic rhinitis with high pollen exposure in grasslands of northern China. Allergy 73, 1232-1243. doi: 10.1111/all.13388

Wang, X. Y., Tian, Z. M., Ning, H. Y., and Wang, X. Y. (2017). Association between airborne pollen distrbution and allergic diseases in Beijing urban area. J. Clin. Otorhinolaryngol. Head Neck Surg. 31, 757-761. doi: 10.13201/j.issn.1001-1781. 2017.10.005
Wishart, D. S. (2016). Emerging applications of metabolomics in drug discovery and precision medicine. Nat. Rev. Drug Discov. 15, 473-484. doi: 10.1038/nrd. 2016.32

Xie, Z. J., Guan, K., and Yin, J. (2019). Advances in the clinical and mechanism research of pollen induced seasonal allergic Asthma. Rev. Am. J. Clin. Exp. Immunol. 8, 1-8.

Xing, Y.-X., Li, M.-H., Tao, L., Ruan, L.-Y., Hong, W., Chen, C., et al. (2018). Anti-cancer effects of emodin on HepG2 cells as revealed by $1 \mathrm{H}$ NMR based metabolic profiling. J. Proteome Res. 17, 1943-1952. doi: 10.1021/acs.jproteome. $8 \mathrm{~b} 00029$

Xu, D., Lv, Y., Wang, J., Yang, M., and Kong, L. (2017). Deciphering the mechanism of Huang-Lian-Jie-Du-Decoction on the treatment of sepsis by formula decomposition and metabolomics: enhancement of cholinergic pathways and inhibition of HMGB-1/TLR4/NF-kappaB signaling. Pharmacol. Res. 121, 94113. doi: 10.1016/j.phrs.2017.04.016

Yasar, H., Kiran, B., Cagatay, T., Ozkul, H., and Icten, S. (2011). The effect of montelukast sodium on serum arginase levels in patients with seasonal allergic rhinitis. Am. J. Rhinol. Allergy 25, e153-e155. doi: 10.2500/ajra.2011.25.3626

Yukselen, A., Kendirli, S. G., Yilmaz, M., Altintas, D. U., and Karakoc, G. B. (2012). Effect of one-year subcutaneous and sublingual immunotherapy on clinical and laboratory parameters in children with rhinitis and asthma: a randomized, placebo-controlled, double-blind, double-dummy study. Int. Arch. Allergy Immunol. 157, 288-298. doi: 10.1159/000327566

Zampieri, M., Sekar, K., Zamboni, N., and Sauer, U. (2017). Frontiers of highthroughput metabolomics. Curr. Opin. Chem. Biol. 36, 15-23. doi: 10.1016/j. cbpa.2016.12.006

Zhang, Q., Wang, J., Liao, S., Li, P., Xu, D., Lv, Y., et al. (2017). Optimization of huang-Lian-Jie-Du-decoction for ischemic stroke treatment and mechanistic study by metabolomic profiling and network analysis. Front. Pharmacol. 8:165. doi: 10.3389/fphar.2017.00165

Zheng, M., Wang, X., and Zhang, L. (2018). Association between allergic and nonallergic rhinitis and obstructive sleep apnea. Curr. Opin. Allergy Clin. Immunol. 18, 16-25. doi: 10.1097/ACI.0000000000000414

Zhuang, Y., Qin, K., Yu, B., Liu, X., Cai, B., and Cai, H. (2018). A metabolomics research based on UHPLC-ESI-Q-TOF-MS coupled with metabolic pathway analysis: treatment effects of stir-frying Xanthii Fructus on allergic rhinitis in mice model. Biomed. Chromatogr. 32:e4352. doi: 10.1002/bmc.4352

Zinkeviciene, A., Kainov, D., Girkontaite, I., Lastauskiene, E., Kvedariene, V., Fu, Y., et al. (2016). Activation of tryptophan and phenylalanine catabolism in the remission phase of allergic contact dermatitis: a pilot study. Int. Arch. Allergy Immunol. 170, 262-268. doi: 10.1159/000450789

Conflict of Interest: The authors declare that the research was conducted in the absence of any commercial or financial relationships that could be construed as a potential conflict of interest.

Copyright (c) 2020 Shi, Pan, Ma, Chen, Yan, Liu, Cao, Huang, Wang, Wang and Wei. This is an open-access article distributed under the terms of the Creative Commons Attribution License (CC BY). The use, distribution or reproduction in other forums is permitted, provided the original author(s) and the copyright owner(s) are credited and that the original publication in this journal is cited, in accordance with accepted academic practice. No use, distribution or reproduction is permitted which does not comply with these terms. 